\title{
Crossover from marginal Fermi liquid to Luttinger liquid behavior in carbon nanotubes
}

\author{
S. Bellucci ${ }^{1}$ and J. González ${ }^{2}$ \\ ${ }^{1}$ INFN-Laboratori Nazionali di Frascati, P. O. Box 13, 00044 Frascati, Italy \\ and INFM-Dipartimento di Fisica, Università di Roma Tor Vergata, Via della Ricerca Scientifica 1, 00133 Roma, Italy \\ ${ }^{2}$ Instituto de Estructura de la Materia. Consejo Superior de Investigaciones Científicas, Serrano 123, 28006 Madrid, Spain
}

(Received 29 June 2001; revised manuscript received 19 September 2001; published 6 November 2001)

\begin{abstract}
We study graphene-based electron systems with long-range Coulomb interaction by performing an analytic continuation in the number of dimensions. We characterize in this way the crossover between the marginal Fermi-liquid behavior of a graphite layer and the Luttinger-liquid behavior at $D=1$. The former persists for any dimension above $D=1$. However, the proximity to the $D=1$ fixed-point strongly influences the phenomenology of quasi-one-dimensional systems, giving rise to an effective power-law behavior of observables like the density of states. This applies to nanotubes of a large radius, for which we predict a lower bound of the corresponding exponent that turns out to be very close to the value measured in multiwalled nanotubes.
\end{abstract}

DOI: 10.1103/PhysRevB.64.201106

PACS number(s): 71.10.Pm, 71.20.Tx, 72.80.Rj

Recently there has been much interest in the search of unconventional electron behavior deviating from the Fermiliquid picture. ${ }^{1}$ Besides this, the other paradigm that is wellestablished on theoretical grounds is the Luttinger-liquid behavior of one-dimensional (1D) electron systems. ${ }^{2,3}$ There have been suggestions that this behavior could be extended to two-dimensional (2D) systems, in the hope that it may explain some of the features of the copper-oxide materials. ${ }^{4}$ However, at least for the Luttinger model, the analytic continuation in the number $D$ of dimensions has shown that the Luttinger-liquid behavior is lost as one departs from $D=1.5,6$

Several authors have also analyzed the possibility that singular interactions could lead to the breakdown of the Fermiliquid picture. ${ }^{7}$ With regard to real low-dimensional systems, such as carbon nanotubes, the main electron interaction comes actually from the long-range Coulomb potential $V(|\mathbf{r}|) \sim 1 /|\mathbf{r}|$. This is also the case of the 2D layers in graphite, which have a vanishing density of states at the Fermi level. Quite remarkably, a quasiparticle decay rate linear in energy has been measured experimentally in graphite, ${ }^{8}$ pointing at the marginal Fermi-liquid behavior in such 2D layers. Due to the singular Coulomb interaction, the imaginary part of the electron self-energy in the 2D system behaves at weak $g$ coupling like $g^{2} \omega .{ }^{9}$ It is crucial, though, the fact that the effective coupling scales at low energy as $g \sim 1 / \log (\omega)$. This prevents the logarithmic suppression of the quasiparticle weight, which gets corrected by terms of order $g^{2} \log (\omega)$ $\sim 1 / \log (\omega) .{ }^{10}$

In this Rapid Communication we investigate whether the long-range Coulomb interaction may lead to the breakdown of the Fermi-liquid behavior at any dimension between $D$ $=1$ and 2 . The issue is significant for the purpose of comparing with recent experimental observations of power-law behavior of the tunneling conductance in multiwalled nanotubes. ${ }^{11}$ These are systems whose description lies between that of a pure $1 \mathrm{D}$ system and the $2 \mathrm{D}$ graphite layer. It turns out, for instance, that the critical exponent measured for tunneling into the bulk of the multiwalled nanotubes is $\alpha \approx 0.3$. This value is close to the exponent found for the single-walled nanotubes. ${ }^{12,13}$ However, it is much larger than expected by taking into account the reduction due to screen- ing $(\sim 1 / \sqrt{N})$ in a wire with a large number $N$ of subbands, what points towards sensible effects of the long-range Coulomb interaction in the system. ${ }^{14}$

We develop the analytic continuation in the number of dimensions having in mind the low-energy modes of metallic nanotubes, which have linear branches crossing at the Fermi level. From this picture, we build at general dimension $D$ a manifold of linear branches in momentum space crossing at a given Fermi point. We consider the Hamiltonian

$$
\begin{aligned}
H= & v_{F} \int_{0}^{\Lambda} d p|\mathbf{p}|^{D-1} \int \frac{d \Omega}{(2 \pi)^{D}} \Psi^{+}(\mathbf{p}) \boldsymbol{\sigma} \cdot \mathbf{p} \Psi(\mathbf{p}) \\
& +e^{2} \int_{0}^{\Lambda} d p|\mathbf{p}|^{D-1} \int \frac{d \Omega}{(2 \pi)^{D}} \rho(\mathbf{p}) \frac{c(D)}{|\mathbf{p}|^{D-1}} \rho(-\mathbf{p}),
\end{aligned}
$$

where the $\sigma_{i}$ matrices are defined formally by $\left\{\sigma_{i}, \sigma_{j}\right\}$ $=2 \delta_{i j}$. Here $\rho(\mathbf{p})$ are density operators made of the electron modes $\Psi(\mathbf{p})$, and $c(D) /|\mathbf{p}|^{D-1}$ corresponds to the Fourier transform of the Coulomb potential in dimension $D$. Its usual logarithmic dependence on $|\mathbf{p}|$ at $D=1$ is obtained by taking the 1D limit with $c(D)=\Gamma((D-1) / 2) /(2 \sqrt{\pi})^{3-D}$.

The dispersion relation $\varepsilon(\mathbf{p})= \pm|\mathbf{p}|$ is that of Dirac fermions, with a vanishing density of states at the Fermi level above $D=1$. This ensures that the Coulomb interaction remains unscreened in the analytic continuation. At $D=2$ we recover the low-energy description of the electronic properties of a graphite layer, dominated by the presence of isolated Fermi points with conical dispersion relation at the corners of the Brillouin zone. ${ }^{15}$

In the above picture, we are neglecting interactions that mix the two inequivalent Fermi points common to the lowenergy spectra of graphite layers and metallic nanotubes. In the latter, such interactions have been considered in Refs. 16 and 17, with the result that they have smaller relative strength $(\sim 0.1 / N$, in terms of the number $N$ of subbands) and remain small down to extremely low energies. More recently, the question has been addressed regarding the interactions in the graphite layer, and it also turns out that phases 
with broken symmetry cannot be realized, unless the system is doped about half-filling ${ }^{18}$ or is in a strong coupling regime. $^{19}$

We implement a differential renormalization group approach, where the integration of high-energy modes at each step leads to corrections to the effective parameters. ${ }^{20}$ Their flow with the cutoff $\Lambda$ determines the fixed points in the limit $\Lambda \rightarrow 0$, which control the physical properties and the universality class to which the model belongs. The present solution overcomes a problem introduced by the transverse scale of the 1D logarithmic potential, which prevents a proper scaling of the model. ${ }^{21}$

We focus on the parameters of the electron self-energy, which get divergent cutoff-dependent corrections. The analysis is simplified by the fact that the polarizability does not have any singular dependence on $\Lambda$ for $D<3$. At $D=1$ this comes from a complete cancellation of self-energy and vertex corrections, as follows from the closed-loop theorem. ${ }^{22}$ In the case of relativistic fermions and $D>1$, one may rely upon the Ward identity that relates the two types of corrections, ${ }^{23}$ to conclude that all the divergent contributions to the polarizability cancel out for $D<3$. As a consequence, the electron charge $e$ stays finite as $\Lambda \rightarrow 0$. The polarizability is then

$$
\Pi\left(\mathbf{k}, \omega_{k}\right)=b(D) \frac{v_{F}^{2-D} \mathbf{k}^{2}}{\left|v_{F}^{2} \mathbf{k}^{2}-\omega_{k}^{2}\right|^{(3-D) / 2}},
$$

where $\quad b(D)=2 / \sqrt{\pi} \Gamma((D+1) / 2)^{2} \Gamma((3-D) / 2) /$ $\left((2 \sqrt{\pi})^{D} \Gamma(D+1)\right)$.

The singular cutoff dependence of the self-energy is given by

$$
\begin{aligned}
\Sigma\left(\mathbf{k}, \omega_{k}\right)= & -e^{2} \int_{0}^{\Lambda} d p|\mathbf{p}|^{D-1} \int \frac{d \Omega}{(2 \pi)^{D}} \int \frac{d \omega_{p}}{2 \pi} \\
& \times G\left(\mathbf{k}-\mathbf{p}, \omega_{k}-\omega_{p}\right) \frac{-i}{\frac{|\mathbf{p}|^{D-1}}{c(D)}+e^{2} \Pi\left(\mathbf{p}, \omega_{p}\right)} .
\end{aligned}
$$

Since we are interested only in the divergent corrections to the cutoff-dependent parameters which determine the fixed points of our solution, we can disregard all finite contributions in (3).

At general $D$, the self-energy (3) shows a logarithmic dependence on the cutoff at small frequency $\omega_{k}$ and small momentum $\mathbf{k}$. This is the signature of the renormalization of the electron field scale and the Fermi velocity. In the low-energy theory with high-energy modes integrated out, the inverse of the electron propagator becomes

$$
\begin{aligned}
\frac{1}{G}= & \frac{1}{G_{0}}-\Sigma \approx Z^{-1}\left(\omega_{k}-v_{F} \boldsymbol{\sigma} \cdot \mathbf{k}\right)-Z^{-1} f(D) \\
& \times \sum_{n=0}^{\infty}(-1)^{n} g^{n+1}\left(\frac{n(3-D)}{n(3-D)+2} \omega_{k}\right. \\
& \left.+\left(1-\frac{2}{D} \frac{n(3-D)+1}{n(3-D)+2}\right) v_{F} \boldsymbol{\sigma} \cdot \mathbf{k}\right) h_{n}(D) \log (\Lambda),
\end{aligned}
$$

where $\quad g=b(D) c(D) e^{2} / v_{F}, \quad f(D)=1 /\left(2^{D} \pi^{(D+1) / 2} \Gamma(D /\right.$ 2) $b(D)), \quad h_{n}(D)=\Gamma(n(3-D) / 2+1 / 2) / \Gamma(n(3-D) / 2+1)$. The quantity $Z^{1 / 2}$ represents the scale of the bare electron field compared to that of the renormalized electron field for which $G$ is computed.

The renormalized propagator $G$ must be cutoffindependent, as it leads to observable quantities in the quantum theory. This condition is enforced by fixing the dependence of the effective parameters $Z$ and $v_{F}$ on $\Lambda$ as more states are integrated out from high-energy shells. We get the differential renormalization group equations

$$
\begin{aligned}
\Lambda \frac{d}{d \Lambda} \log Z(\Lambda)= & -f(D) \sum_{n=0}^{\infty}(-1)^{n} g^{n+1} \\
& \times \frac{n(3-D)}{n(3-D)+2} h_{n}(D), \\
\Lambda \frac{d}{d \Lambda} v_{F}(\Lambda)= & -v_{F} f(D) \sum_{n=0}^{\infty}(-1)^{n} g^{n+1} \\
& \times\left(1-\frac{1}{D} \frac{n(3-D)(2-D)+2}{n(3-D)+2}\right) h_{n}(D) .
\end{aligned}
$$

At $D=2$, the right-hand side of these equations can be summed up to the functions that have been found previously in the renormalization of Dirac fermions with Coulomb interaction. ${ }^{10}$ Furthermore, they also provide meaningful expressions in the $1 \mathrm{D}$ limit. At $D=1$, the right-hand side of Eq. (6) vanishes identically as a function of the variable $g$. Therefore, the 1D model has formally a line of fixed points, as it happens in the case of a short-range interaction. The scaling of the electron wave function can be read from the right-hand side of Eq. (5), which becomes (2 $+g) /(2 \sqrt{1+g})-1$ at $D=1$. This coincides with the anomalous dimension that is found in the solution of the Luttinger model, what provides an independent check of the renormalization group approach to the 1D system.

We therefore have a model that interpolates between marginal Fermi-liquid behavior, that is known to characterize the 2D model, and non-Fermi-liquid behavior at $D=1$. As the electron charge $e$ is not renormalized for $D<3$, the scaling of the effective coupling $g=b(D) c(D) e^{2} / v_{F}$ is given after Eq. (6) by

$$
\begin{aligned}
\Lambda \frac{d}{d \Lambda} g(\Lambda)= & f(D) \sum_{n=0}^{\infty}(-1)^{n} g^{n+2} \\
& \times\left(1-\frac{1}{D} \frac{n(3-D)(2-D)+2}{n(3-D)+2}\right) h_{n}(D) .
\end{aligned}
$$

The right-hand side of Eq. (7) is a monotonous increasing function of $g$, for any dimension between 1 and 2, as observed in Fig. 1.

We find that, away from $D=1$, there is only one fixed point of the renormalization group at $g=0$. The scale dependence of the effective coupling $e^{2} / v_{F}$ is displayed for differ- 


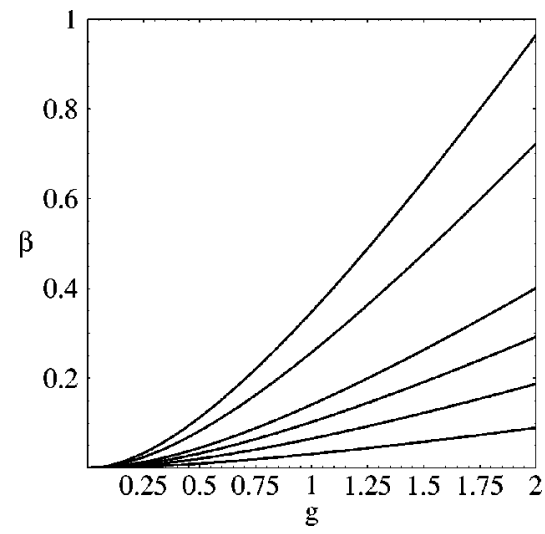

FIG. 1. Plot of the $\beta$ function at the right-hand side of Eq. (7) for different dimensions. From top to bottom, the curves correspond to the values $D=2.0,1.7,1.4,1.3,1.2$, and 1.1 .

ent values of $D$ in Fig. 2, where the flow to the fixed point is seen. Consequently, the scale $Z$ of the wave function is not renormalized to zero in the low-energy limit, and the quasiparticle weight remains finite above $D=1$. We conclude then that, even in a model that keeps the Coulomb interaction unscreened, the breakdown of the Fermi-liquid behavior only takes place formally at $D=1$.

The subtlety concerning the long-range Coulomb interaction is that the function $c(D)$ diverges in the limit $D \rightarrow 1$. This is actually what transforms the power-law dependence of the potential into a logarithmic dependence at $D=1$. We observe that the 1D limit and the low-energy limit $\Lambda \rightarrow 0$ do not commute. If we stick to $D=1$, we obtain a divergent coupling $g$ for the Coulomb interaction as well as a divergent electron scaling dimension. At any dimension slightly above $D=1$, however, the fixed point is at $g=0$, with its corresponding vanishing anomalous dimension.

In order to understand whether the 1D model has any stable fixed point for finite values of $e^{2} / v_{F}$, one can study the model by performing an expansion in powers of $g^{-1}$. The Fermi velocity is renormalized by terms that are analytic near the point $g=\infty$, and that lead to the scaling equation

$$
\Lambda \frac{d}{d \Lambda} v_{F}(\Lambda)=v_{F} f(D)\left(3-D-\frac{2}{D}\right) \frac{\Gamma(D / 2-1)}{\Gamma((D+1) / 2)}
$$

up to terms of order $O\left(g^{-1}\right)$. In the limit $D \rightarrow 1, g \rightarrow \infty$, the

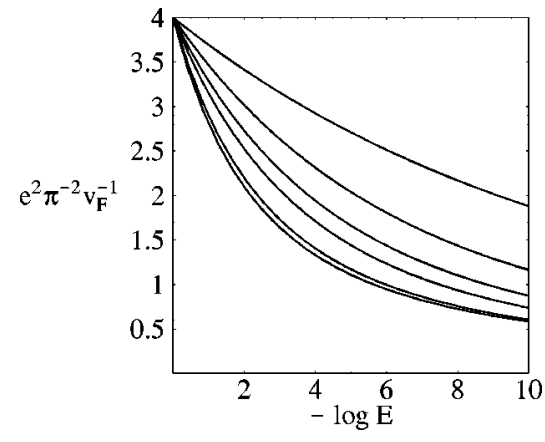

FIG. 2. From top to bottom, curves of the effective coupling as a function of the energy scale for $D=1.1,1.2,1.3,1.4,1.7$, and 2.0.

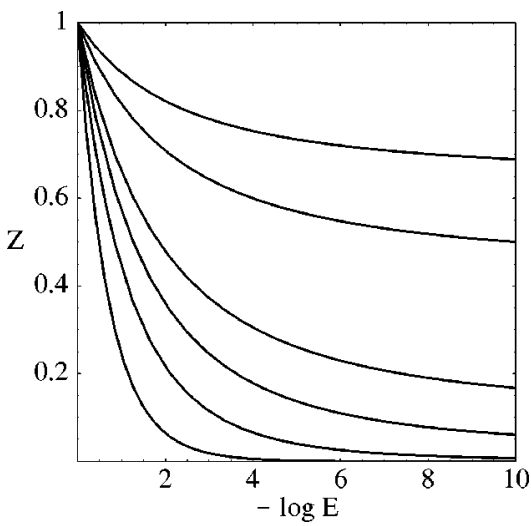

FIG. 3. Plot of the electron field scale $Z$ for a value of the bare coupling $e^{2} /\left(\pi^{2} v_{F}\right)=4.0$. The different curves correspond, from top to bottom, to $D=2.0,1.7,1.4,1.3,1.2$, and 1.1 .

right-hand side of Eq. (8) vanishes identically. This confirms, on nonperturbative grounds, that the 1D model with the Coulomb interaction has a line of fixed points covering all values of $e^{2} / v_{F}$.

In the vicinity of $D=1$, the presence of such critical line becomes sensible, and a crossover takes place to a behavior with a sharp reduction of the quasiparticle weight. This can be seen in the renormalization of the electron field scale $Z$, displayed in Fig. 3. For values of $D$ above $\approx 1.2$, we have a clear signature of quasiparticles in the value of $Z$ at low energies. For lower values of $D$, the picture cannot be distinguished from that of a vanishing quasiparticle weight for all practical purposes.

The above picture allows us to make contact with the experiments carried out in multiwalled nanotubes. ${ }^{11}$ In the proximity of the $D=1$ fixed point, the density of states displays an effective power-law behavior, with an increasingly large exponent. Moving to the other side of the crossover, the density of states approaches the well-known behavior of the graphite layer, $n(\varepsilon) \sim|\varepsilon|$. In Fig. 4 we give the representation of the density of states

$$
n(\varepsilon) \sim Z(\varepsilon)|\varepsilon|^{D-1}
$$

for several dimensions approaching $D=1$.

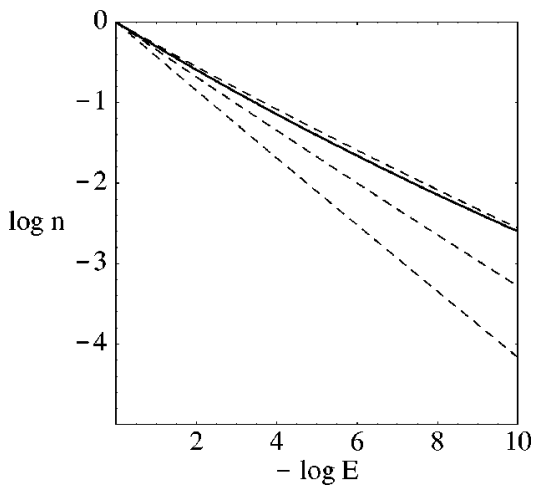

FIG. 4. Plot of the logarithm of the density of states for a value of the bare coupling $e^{2} /\left(\pi^{2} v_{F}\right)=0.5$. The solid line corresponds to $D=1.1$ and the dashed lines correspond, from top to bottom, to $D$ $=1.2,1.3$, and 1.4. 
A value $e^{2} /\left(\pi^{2} v_{F}\right)=0.5$ for the bare coupling is appropriate for typical multiwalled nanotubes, as it takes into account the reduction due to the interaction with the inner metallic cylinders. ${ }^{24}$ We observe that the exponents of $n(\varepsilon)$ at different dimensions are always larger than a lower bound $\alpha \approx 0.26$. This is in agreement with the values measured experimentally. Our analysis stresses the need of an appropriate description of the dimensional crossover between one and two dimensions, showing that the picture of a thick nanotube as an aggregate of 1D channels does not allow to obtain the correct values of the critical exponents.

To summarize, we have studied the renormalization of the Coulomb interaction in graphene-based structures. We have made a rigorous characterization of the different behaviors, as we have proceeded by identifying the fixed points of the theory. We have seen that the Fermi-liquid behavior persists formally for any dimension above $D=1$, as it also happens in the case of a short-range interaction. ${ }^{5}$ On the other hand, the proximity to the 1D fixed point influences strongly the phenomenology of real quasi-one-dimensional systems, giving rise to an effective power-law behavior of observables like the tunneling density of states. This is the case of the multiwalled nanotubes, for which we predict a lower bound for the corresponding exponent that turns out to be very close to the value measured experimentally.

Financial support from CICyT (Spain) and CAM (Madrid, Spain) through Grant Nos. PB96/0875 and 07N/0045/98 is gratefully acknowledged.
${ }^{1}$ J. Gan and E. Wong, Phys. Rev. Lett. 71, 4226 (1993); C. Nayak and F. Wilczek, Nucl. Phys. B 417, 359 (1994); S. Chakravarty, R.E. Norton, and O.F. Syljuasen, Phys. Rev. Lett. 74, 1423 (1995); J. González, F. Guinea, and M.A.H. Vozmediano, Nucl. Phys. B 485, 694 (1997).

${ }^{2}$ J. Sólyom, Adv. Phys. 28, 201 (1979).

${ }^{3}$ F.D.M. Haldane, J. Phys. C 14, 2585 (1981).

${ }^{4}$ P.W. Anderson, The Theory of Superconductivity in the High-T Cuprates (Princeton University Press, Princeton, 1997).

${ }^{5}$ C. Castellani, C. Di Castro, and W. Metzner, Phys. Rev. Lett. 72, 316 (1994).

${ }^{6}$ This result is reinforced by the fact that the coupling of Luttinger liquids by the slightest hopping in the transverse direction leads also to Fermi liquid behavior of the system, as shown by X.G. Wen, Phys. Rev. B 42, 6623 (1990); E. Arrigoni, Phys. Rev. Lett. 83, 128 (1999).

${ }^{7}$ P.-A. Bares and X.G. Wen, Phys. Rev. B 48, 8636 (1993); A. Houghton, H.-J. Kwon, J.B. Marston, and R. Shankar, J. Phys.: Condens. Matter 6, 4909 (1994); C. Castellani, C. Di Castro, and A. Maccarone, Phys. Rev. B 55, 2676 (1997).

${ }^{8}$ S. Yu et al., Phys. Rev. Lett. 76, 483 (1996).

${ }^{9}$ J. González, F. Guinea, and M.A.H. Vozmediano, Phys. Rev. Lett. 77, 3589 (1996).

${ }^{10}$ J. González, F. Guinea, and M.A.H. Vozmediano, Phys. Rev. B 59, R2474 (1999).

${ }^{11}$ A. Bachtold, Phys. Rev. Lett. 87, 166801 (2001).
${ }^{12}$ M. Bockrath et al., Nature (London) 397, 598 (1999).

${ }^{13}$ Z. Yao et al., Nature (London) 402, 273 (1999).

${ }^{14}$ See also R. Egger and A. O. Gogolin, Phys. Rev. Lett. 87, 066401 (2001).

${ }^{15}$ J. González, F. Guinea, and M.A.H. Vozmediano, Nucl. Phys. B 406, 771 (1993).

${ }^{16}$ L. Balents and M.P.A. Fisher, Phys. Rev. B 55, R11 973 (1997).

${ }^{17}$ R. Egger and A.O. Gogolin, Phys. Rev. Lett. 79, 5082 (1997); Eur. Phys. J. B 3, 281 (1998).

${ }^{18}$ J. González, F. Guinea, and M.A.H. Vozmediano, Phys. Rev. B 63, 134421 (2001).

${ }^{19}$ D.V. Khveshchenko, report cond-mat/0101306.

${ }^{20}$ R. Shankar, Rev. Mod. Phys. 66, 129 (1994).

${ }^{21}$ On phenomenological grounds, one can however make sense of such dependence on the transverse dimension, which leads to scale-dependent critical exponents as reported by C. Kane, L. Balents, and M.P.A. Fisher, Phys. Rev. Lett. 79, 5086 (1997); S. Bellucci and J. González, Eur. Phys. J. B 18, 3 (2000); D. W. Wang, A. J. Millis, and S. Das Sarma, Phys. Rev. B 64, 193307 (2001).

${ }^{22}$ P. Kopietz, J. Hermisson, and K. Schönhammer, Phys. Rev. B 52, 10877 (1995); W. Metzner, C. Castellani, and C. Di Castro, Adv. Phys. 47, 317 (1998).

${ }^{23}$ C. Itzykson and B. Zuber, Quantum Field Theory (McGraw-Hill, New York, 1980).

${ }^{24}$ R. Egger, Phys. Rev. Lett. 83, 5547 (1999). 